\title{
Mobile Medicine: Can Emerging Mobile Technologies Enable Patient-Oriented Medicine?
}

This is the first special issue of the Annals of Biomedical Engineering dedicated to highlighting the latest academic innovations and interdisciplinary efforts towards establishing emerging mobile-based medical platforms. The rapid development of mobile electronic devices and associated software applications has been significantly reshaping the way that people share information and interact with each other universally. Recently, this emerging technological trend has started offering new innovative opportunities to improve personal health and healthcare systems which may lead to drastic changes in how medical care will be delivered worldwide in the foreseeable future. Notably, both growing elderly populations and rising rates of chronic diseases keep challenging the overburdened healthcare systems in developed countries, while in the developing world shortages of basic medical services present a major concern. This emerging field, collectively known as mobile medicine, aims to (a) allow patients to selfmonitor their health conditions on a continuous basis, (b) provide doctors with real-time access to patients' health data, (c) facilitate communication and information sharing between the two parties, and (d) generate individual health data sets useful for future personalized medicine, which is becoming a very attractive alternative to conventional medicine. It is important to note that this unprecedented technological revolution has been enabled and accelerated by multidisciplinary efforts from bioengineering, clinical medicine, bioinformatics, microelectronics, microelectromechanical systems, microfluidics, communications, cloud computing, and others.

This special issue of ABME is dedicated to a wide range of research articles at the frontier of mobilebased healthcare and medical solutions, covering topics such as wearable microsensors, portable pointof-care instruments, cellphone-based detection methods, and lab-on-a-chip analytical devices. Despite many developments and enormous progress, a number of technical challenges, such as sensitive reliable detection, flexible human-sensor interfaces, signal processing and data accuracy, have limited the wide-scale adoption of mobile medical approaches. These problems are being actively researched and some of them have been addressed by the articles in this Special Issue of Mobile Medicine.
Specifically, the topics of this issue include an article by Lillehoj and colleagues reviewing recent advancements and developments in smartphone-based pointof-care systems, comprised of detectors, sample processors, disposable chips, batteries and software, with an emphasis on cell and biomolecular detection for mobile medical applications. Park and colleagues summarize the latest technological developments and applications of electrocardiogram (ECG) recordings using non-contact wearable capacitive electrodes. A wearable surgical navigation system has been proposed by $\mathrm{Xu}$ and colleagues, combining the sensitivity and specificity of a fluorescence imaging system and

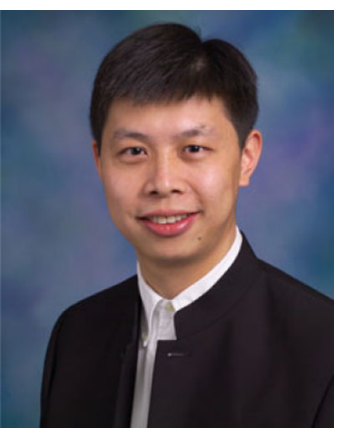

Tingrui Pan.

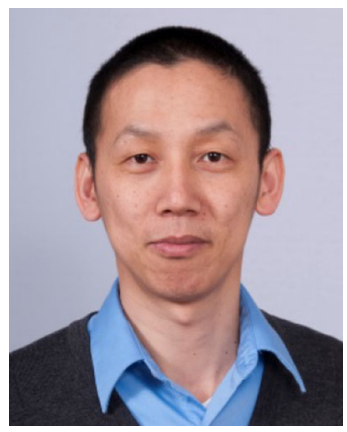

Yong Xu. the mobility of a wearable Google Glass for real-time intraoperative imaging of the surgical margin in cancer resection surgery. Chon and colleagues investigate algorithms to improve photoplethysmogram (PPG) signals by reducing motion artifacts for real-time monitoring of vital signals. To utilize state-of-the-art solid-state sensing technologies, $\mathrm{Xu}$ and colleagues review the applications of accelerometers in the detection of body acoustic signals for vital physiological and pathological information in mobile healthcare. As an alternative sensing scheme, Pan and colleagues introduce a miniature, flexible, transparent, highly sensitive and wearable pressure sensor with embedded microfluidic elements, which has been utilized to perform arterial tonometry with the capability of noninvasive monitoring of arterial blood pressure waveforms in a realtime and continuous fashion. In the active area of development of lab-on-a-chip point-of-care systems, Shin and Wang review recent developments in the use 
of magnetically actuated droplets in point-of-care molecular diagnostic platforms for biological samples. Furthermore, an integrated point-of-care flow cytometry device proposed and investigated by Huang and colleagues employs a microfluidic approach to analyze human blood profiles for cell counting and disease diagnosis. Wong and colleagues report a rapid testing method of antimicrobial susceptibility combining the phenotypic culture of bacterial pathogens in physiological samples and electrochemical sensing of specific strains for rapid diagnosis of bacterial infection in nontraditional healthcare settings. In addition, Kamei and colleagues report the utilization of dextran-coated gold nanoprobes as a colorimetric indicator for lateralflow immunoassays in point-of-care detection. Zheng and colleagues conclude the issue with an overview of progress on the development of integrated lab-on-chip devices and systems for point-of-care blood plasma analysis in viral infectious diseases.

It has been anticipated that the patient-oriented medical approach could eventually lead to improvements in the efficiency and accuracy of how medicine is delivered while significantly reducing its cost and time. Transformative ideas presented in this issue along with many others will keep providing the field of mobile medicine with innovative and exciting directions. We hope that this Special Issue can serve as a guide on various active topics of research for researchers and students, and inspire further interest and innovation in this promising field.

\section{Tingrui PAN}

Biomedical Engineering

University of California, Davis, CA, USA

Electronic mail: tingrui@ucdavis.edu

Yong Xu

Electrical and Computer Engineering, Wayne State University, Detroit, MI, USA 